\title{
RHINO-an autonomous interactive surveillance robot for the needed ones: design and study case.
}

\author{
Michel Ochoa ${ }^{1}$, Gabriel Aguiar ${ }^{1}$ and Andrés Erazo ${ }^{2}$ \\ ${ }^{1}$ Universidad de las Fuerzas Armadas - ESPE, Department of Science of Energy and Mechanics, 170501Av. Gral. Rumiñahui, Ecuador \\ ${ }^{2}$ Universidad de las Fuerzas Armadas - ESPE, Department of Electrical and Electronic Engineering, 170501 Av. Gral. Rumiñahui, Ecuador
}

\begin{abstract}
Healthcare services are in need of new tools and gadgets that could provide surveillance and people interaction of the needed ones with their surrounding environment. In order to address this problematic, an autonomous-interactive surveillance robot system is proposed in this research. RHINO (Robotic-Human Interface for the Needed Ones) was designed under particular normative and will provide commonly needed features in the healthcare field. One of the principal aspects of the device is its particular design which allows the patient to have some privacy under a surveilled environment and without causing any interference in their daily lives. Another main factor is the ability of notifying the care provider or the family of the patient with e-mail or text-sms notifications in case of abnormal activities; so that video access by provider or Skype calls by family relatives could be performed throughout RHINO.
\end{abstract}

\section{Introduction}

The surveillance of the needed ones and their interaction with people is a subtle topic for health care providers and families. The normative regulates the use of methods, technologies and applications in order to give best quality to these services. If we talk about surveillance, there are several different types of technology equipment that can be used for surveillance of the needed ones [1]. The most important are:

- Video-monitoring: it is possible to implement color audio-video communications in real-time for surveillance of the individual being monitored, but it is necessary that the care provider checks the video constantly.

- Health monitors: they are the size of a watch with a continuous pulse, skin, temperature and movement monitoring. Also, an alarm is transmitted in case of an abnormal activity.

- Fall detectors: there are wearable mechanisms that detect an impact greater than a certain threshold and send a notification.

In addition, there are hip protectors, pressure mats, door alerts, movement detectors, and others.

Limitations of these systems are the lack of autonomy and the need of the care provider to check constantly the equipment, and the patient. Robots can solve these problems, since they have the ability to interact with humans in their daily life. In a research in Osaka University, an English-speaker robot was used for interacting and teaching language to first and sixth level learners. The trial took place for the period of two weeks and the results proved that the children showed interest in interacting with the robot and their English-speaking abilities were increased during the trial time [2].

Robots are accepted entities in the medical fields and new methods to improve their functionality are developed; like ROS, a complete Open Source Operative System to control robots with all the tools and features a personal desktop could provide. Under this scope, the current research shows how an autonomous-interactive surveillance system could be incorporated into a robot for taking care of the needed ones. RHINO (Robotic-Human Interface for the Needed Ones) is a new technology, which follows the tendency of medical robots bringing services of care and interaction with patients.

\section{Related Works}

Nowadays, home accidents are a public health problem. Home accidents are responsible for the $5 \%$ of deaths in children aged 5 years or younger in Latin America [3]. However, the statistics don't show the actual number of people injured from home accidents, where we have between 200 and 1200 injuries per death. Ninety per cent of these accidents could have been prevented if there have been adult supervision [4].Home accidents are also a big problem between the elderly, researches indicate that a third of people aged 65 and older fall down each year, being half of them not just one but multiple falls. A lot of these falls are related with hospitalization, institutionalization, hip fractures, fear of falling, mortality and other consequences [5].

As a result of previous researches [2, 6], surveillance interactive robot has been proposed as a 
possible solution for helping, monitoring, and assisting people without the necessity of institutionalize them or to hire health care personnel. Companion robots can increase the positive mood in elderly living in nursing homes by decreasing levels of stress and loneliness, and increasing communication activity with others [6]. Currently, there are countless of companionship robots, four of the most relevant are PARO, PEARL, ICAT and AIBO [7]-[11](Figure 1).

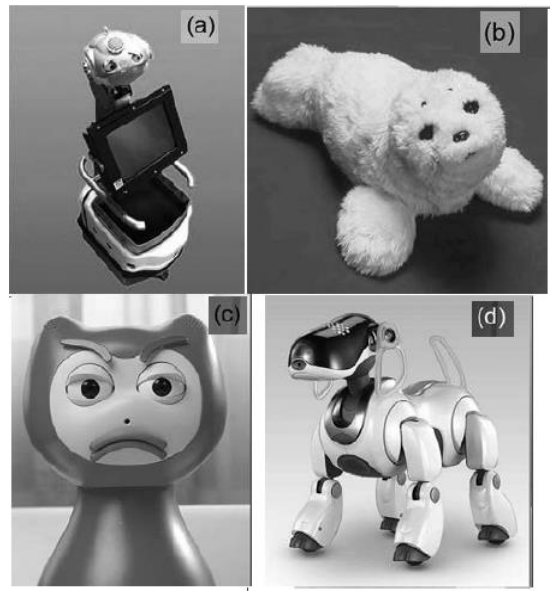

Figure 1.Companion and assisting robots $[7,8,10,11]$.

The most used configuration for smart robots, nomad scouts and assistive robots is the two-wheeled centered differential drive with third point of contact [12].

Some of the previous technologies described use tracking as their core system or component. Tracking gives to autonomous system the ability to interact with their environment. Human tracking algorithm [13]-[16] become the basis for human-robot interactions and their possible applications are uncountable [17].

Stationary results of the human tracking are reliable [18]-[20]. However, if the camera is tracking from a moving platform, those methods tend to fail. Choi et al indicates several approaches that aboard this problem [18]. In addition, the study proposes a general framework for tracking multiple people from a moving camera, performing data association to track people, and estimating the camera motion. Other alternatives, like adaptive HOG human tracker, upgraded by OpenCV libraries have proven their validity [18].

Choosing the camera for making the tracking cannot be taken lightly. A proper description of the use of stereo and monocular cameras for mapping in mobile platforms is developed at [21]. The use of RGB-D cameras such as the Microsoft Kinect, are good alternatives for capturing RGB images along with perpixel depth information, mapping and recognition applications [21] [22]. Random errors of depth measurements are minimums when data is acquired within 1-3 meters of distance to the sensor [22].

Nowadays robots application had been improved by Internet standard communications. This communication techniques enable features as telecare and remote surveillance to a new level [23].

Most of autonomous surveillance systems are not capable of sending notifications, e-mails or text messages to the care providers. Such notification will inform anomalies detected in the surveillance. This tool will keep the care provider notified at all time, avoiding constant monitoring [24]. But a complete and limited autonomy from the surveillance system could raise ethical issues. In the work developed by A. Sharkey \& N. Sharkey, they discuss main ethical concerns associated with robot care for the elderly. They explain that all autonomous surveillance systems should have their "off switch" [25].

The straightforward remote control applications used for accessing any system from a distance, not only reduce the autonomy of the system, they also allow the care provider to control the mobile robot and access the information collected from anywhere through the internet. The benefits of remote control application are that they provide a secure connection and environment, plus a register of all the times care providers have accessed to the surveillance system.

The work of care providers is very emotional and physical demanding. It can even affect the family members causing them burden and stress. The investigation held by J. Czaja \& P. Rubert describe how technology can be used to provide support to care providers, increasing the quality of life for both, care providers and care recipients[17].

Technological developments focused on the care and surveillance of needed people, must consider fundamental care standards. Fundamental standards as person-centered care, dignity and respect, consent, safety, among others; should be of our concern when we perform innovation of any kind [26].

There are some organizations that monitors, review and regulate care services to make sure they meet this fundamental standard of quality and safety [27]. The independent regulator of health and social care in England and worldwide recognized, The Care Quality Commission (CQC), establishes a normative for using surveillance. This normative gives some guidelines for any technological developments in this field. In addition, there are some legal obligations under the Human Rights Act of 1998 and the Regulation of Investigatory Powers Act of 2000 (RIPA) that providers should be aware of [28].

The CQC advises that covert surveillance must be limited both in time and in purpose to remain lawful. Also, the provider must assure that only authorized people can access the information collected. The use of an autonomous and independent surveillance system, capable of detecting anomalies in the behavior of the individual being monitored, ensures the legal right of privacy of the individual. Furthermore, these systems must secure the information collected and guarantee that only authorized people access to this service. Finally, a record of when and why recorded information has been accessed could be generated by these systems. All of this will ensure the correct use of the critical information providers have to deal with.

\section{RHINO Prototype}


RHINO is an autonomous interactive surveillance system that allows the care provider to monitor the subject in real time(Figure 2). RHINO is a mobile robot that has two operating modes:

- Autonomous mode: the robot supervises the person being monitored using a computer vision algorithm and a pyroelectric infrared sensor. If an anomaly occurs, the robot could send a notification (mail or sms) to the care provider (Figure 3d).

- Control mode: the care provider could call the person been monitored using video calls and he could be able to control the robot from any place with internet access, allowing an interaction between them (Figure 3a and 3c).

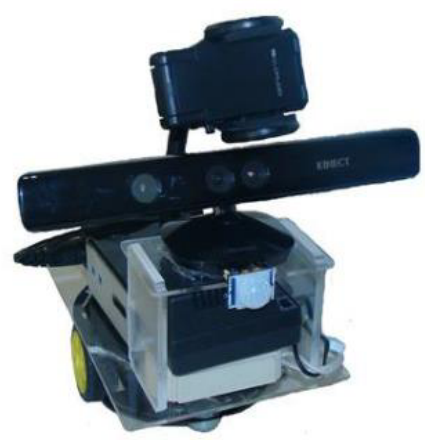

Figure 2.RHINO prototype

The robot was armed on a two-wheeled centered differential drive with a third point of contact. The platform was armed using commercial elements, because is the first prototype of the RHINO models.

The robot uses two DC motors that are controlled by a microcontroller board that is connected to the master controller. The master node is an Intel NUC with Ubuntu 14 for its operating system. The motors are connected to an H-bridge for switching their spinning direction and each motor has an incremental encoder to control the displacement of each wheel during the movement of the robot.

The algorithm was implemented in ROS Indigo using node programming and communication by messages. The ROS node connects to the microcontroller board, and using serial communication, the master node sends a message telling the board that the robot should perform a movement or an action. For the control mode, a ROS node was used. This node allows the care provider to use a key of the keyboard storing it as a char variable and sent via serial port to the microcontroller board using the serial communication node of ROS (Rosserial_python). The variable will be validated in the microcontroller board, and depending of the validation, the robot will move forward, backward, right, left or stop(Figure 3a).

The autonomy of the RHINO's surveillance system is based on the detection and tracking of the individual being monitored (Figure 3b). Without this, RHINO will not be capable of monitoring the individual. In the previous section we discuss the advantages of using an RGB-D camera. RHINO is enhanced with a Microsoft Kinect camera and a human tracking method for mobile platforms. It also counts with an adaptive HOG human tracker, coded using OpenCV features.
Tracking will provide the position of the individual in an $\mathrm{x}, \mathrm{y}$ and $\mathrm{z}$ system regarding the camera position, and therefore regarding the position of the robot. A secure zone is established to reduce the maximum possible intrusion of RHINO to the individual being monitored. If the individual is getting away from the robot around 2 meters, the robot will follow the subject; if not, RHINO will stay still monitoring the individual. A python subscriber receives the position data, process it and control the motor movement through serial communication.

Notifications to the care provider of an abnormal parameter, such as the lack of movement, is processed by a PIR sensor algorithm. The PIR sensor would be constantly sending high pulses while there is movement, and if the high pulses are not received by the microcontroller board, a flag via serial communication will be sent to a ROS node. After receiving the flag, the ROS node would subscribe to another node call pushing box which is connected to an internet cloud that can send notifications based on API calls. This cloud was previously configured with the service needed (e-mail, notify my android, prowl, etc.) and the scenario (sending a notification with the abnormal parameter, "lack of movement").

This autonomy is the key to provide the individual being monitored with a more truly privacy, without the need of a care provider always looking after the subject. And even more, all the connection activity is reported in a .txt document automatically. This is useful and important because this register is a key point of the care quality surveillance normative.

If the care provider or the family of the individual being monitored wants to communicate with the subject, they can use Skype to start a video call and RHINO will automatically answer it. To ensure privacy, it will be needed a password to establish the communication.

Skype's text, voice and video make it simple to connect people and stay in touch with them wherever you are. Boulos et al in their study of sociable technologies in health and health care education remarks the potential of Skype, and mention these technologies should not be underestimated. Features like automatic answering and passcode for accessing calls provide a valuable and secure use for care surveillance application. Also, the study affirms: "Sociable interactive technologies have the potential to augment patients' and older peoples' sense of belonging to a supportive kindred community, thereby reducing their social isolation" [29].

TeamViewer is the remote desktop that will control the autonomy of RHINO. The care provider can access RHINO through this application in his cell phone, tablet or PC. This provides the ability to turn off or on RHINO and access data recorded like PIR events and video. This is also the interface to control the robot from anywhere through the internet. For example, if the care provider calls the individual using Skype, he can control the movement of RHINO to interact with the individual in a more unique way. 


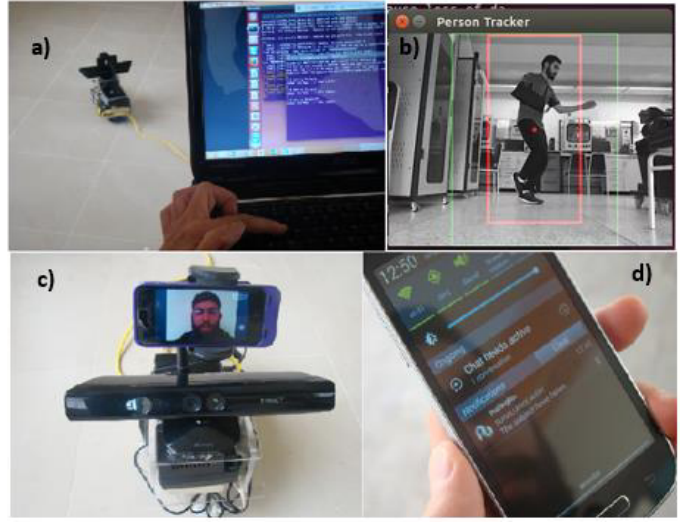

Figure 3:RHINO features; a) Control mode, b) Autonomous mode, c) RHINO interaction and d) Cell phone notification.(https://www.youtube.com/channel/UC3qG3bWFBaHf7Ol_UI4X3g)

\section{Results and Discussion}

A Proof of Concept (POC) was conducted with potential users. These users have been people with someone that need surveillance and have experience with traditional surveillance systems, like doctors, medical interns and family of needed ones. The purpose of this POC was to identify how care provider's responds to this autonomous surveillance system. We describe the conceptual features to our potential users; and using video tools, we showed them the results obtained. A survey was conducted at the end of the POC (Figure 4).
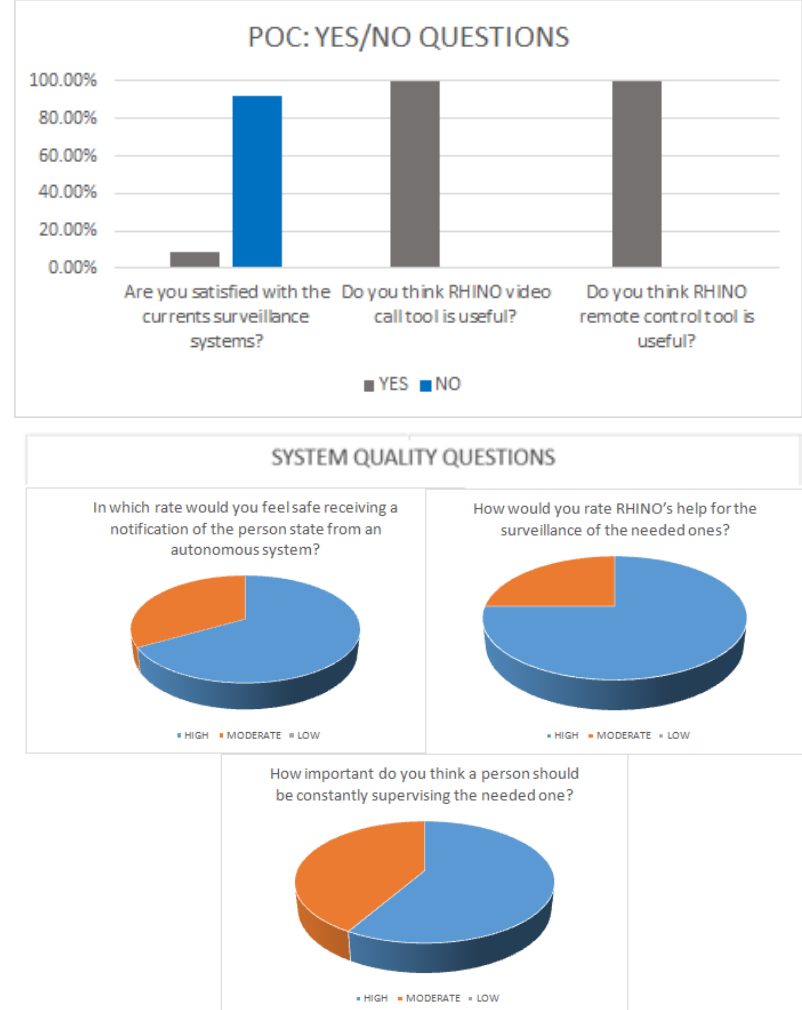

Figure 4:POC: Yes/No Questions and System Quality Questions.

The results obtained from this POC shows us that $92.31 \%$ of the respondents are not satisfied with the current and traditional surveillance system. It is considered that new systems should provide more liberty to care providers and individuals being monitored, without reducing the control and care. The results also revealed that $53.85 \%$ of the research participants considered really important a permanent surveillance of patients. However and in contrast, $46.15 \%$ of them presented a tendency to avoid permanent surveillance as an important factor. This second group could be related to the problematic of privacy and intrusive factor that our system deal with to provide a better service. Another important value to take into account is that more than $61.54 \%$ of the polled consider useful the alert notifies RHINO provided. In the same way and with almost the same percentage, the $69.23 \%$ of the participants approved and rated RHINO as a trusty autonomous surveillance system. In addition, RHINO was accepted as a more personalized way of surveillance for the loved ones. Two aspects of the system were checked by the respondents under this scope: video calls and teleoperation mode; and both tools were $100 \%$ accepted.

Under the same perspective, measures taken to ensure the security of the system, like the passcode for the video calls and the password for the remote control of RHINO, fulfill their purpose. The potential users considered that this aspect is important and was very well implemented.

The tracking algorithm has to deal with noises and occlusion from the Kinect. The uses of filters, like the Kalman filter, have reported good results; reducing noise and estimating with good accuracy human's motion detection in mobile robots [30] [31].Further investigations are needed to incorporate advanced features for medical and health care. One of the future improvement includes the implementation of a health monitor bracelet that will measure pulse, temperature and other important parameters and would transfer these data to the master node; which in turn will process this information and send a notification if there is any abnormality [1].

The approval RHINO received from care providers during the proof of concept demonstrated the growth of the acceptance of robots at healthcare services and facilities. This approval motivates us in developing new systems for care and surveillance for the needed ones. However, innovations not only should focus on the control of the patient and we expect that further versions of RHINO help patients regain their confidence in order to help them in a process of reintegration to the society. This will only be achieved throughout a more robust human robot interaction system, that could establish a truly bound of trust with the patient.

There has always been a constant debate between privacy rights and the need of complete surveillance [28]. Individuals that suffer from dementia or complete body paralysis must be under surveillance all the time. The only way these individuals could regain some of their privacy rights is changing the traditional surveillance methods to those who are autonomous. Surveillance made by a person is always intrusive; on the other hand, systems that doesn't require the control of a person to fulfill their main objective, give privacy and control care to individuals under surveillance [28]. 


\section{Conclusions and Future Works}

RHINO is the first prototype of RHINO models, which is why in our research we found some benefits and also some setbacks. ROS worked perfectly like a master node, allowing the care provider to control all nodes in the robot, and allowing the communications between them.

Also, the human tracking system, based on the Microsoft Kinect and OpenCV features, was able to complete the objective established for a noninvasive surveillance. Furthermore, the Node in charge of the lack of movement worked as expected, sending the right notification as needed. In parallel, the remote control provided by TeamViewer offers an exceptional communication between the care provider and the robot. In resume, this first prototype of the RHINO models proved that it is possible to develop surveillance systems that respect fundamental care standards and it is capable of performing care services.

Finally, the autonomy of RHINO accomplishes his main objective, reducing privacy invasion. POC results corroborate that this project respects the right of privacy of the individual being monitored to a greater degree than conventional methods. RHINO is the tool of the future, a health care monitoring system which respect your privacy and helps you when you are in need of your doctor, of your family or just of the attention of any of them.

\section{References}

1. F. G. Miskelly, Assistive technology in elderly care, Age Ageing, 30, pp.455-458 (2001)

2. C. Trial, T. Hiranoet al, Interactive Robots as Social Partners and Peer Tutors for childrens: A field trial,HCI, 19, pp.61-84 (2004)

3. A. Benavides-Lara \& M. Vargas-Salas, Original Mortality by accidental causes in children of 5 years and younger at the "Nacional de Niños" Hospital, Acta Med. Costarric., 50, pp.22-28 (2008)

4. Y. Benguigui, J.C Bossio\& H. Fernández, Operations Research on Integrated Management to Prevalent Childhood Illness, OPS/OMS, pp.437-532 (2001)

5. C. a Reyes-Ortiz, S. Al Snih, \& K. S. Markides, Falls among elderly persons in Latin America and the Caribbean and among elderly MexicanAmericans, RPSP/PAJPH, 17, pp.362-9 (2005)

6. J. Broekens, M. Heerink, \& H. Rosendal, Assistive social robots in elderly care: a review, Gerontechnology, 8, pp.94-103(2009)

7. M. Pollack et al, Pearl: A mobile robotic assistant for the elderly, AAAI, pp.85-91, (2002)

8. K. Wada et al,Effects of robot assisted activity to elderly people who stay at a health service facility for the aged,IROS,3, pp.2847-2852 (2003)

9. K. Wadaet al, Effects of robot-assisted activity for elderly people and nurses at a day service center, IEEE, 92, pp.1780-1788 (2004)

10. A. Van Breemen, X. Yan \& B. Meerbeek, iCat: an animated user-interface robot with personality, AAMAS, pp.143-144 (2005)
11. M. Fujita, AIBO: Toward the era of digital creatures, IJRR, 20, pp.781-794 (2001)

12. R. Siegwart\& I. R. Nourbakhsh, Introduction to Autonomous Mobile Robots, p. 17, 30, 35 (2006)

13. O. Tuzel, F. Porikli, \& P. Meer, Human detection via classification on riemannian manifolds, CVPR'07, pp.1-8 (2007)

14. V. Ferrari, M. Marin-Jimenez, \& A. Zisserman, Progressive search space reduction for human pose estimation, CVPR'08, pp.1-8 (2008)

15. P. Felzenszwalb, R. Girshick et al, Object detection with discriminatively trained part based models, PAMI, 32, pp.1627-1645 (2010)

16. N. Dalal\& B. Triggs, Histograms of oriented gradients for human detection, CVPR, 1, pp. 886893 (2005)

17. S. Czaja\& M. Rubert, Telecommunications technology as an aid to family caregivers of persons with dementia, Psychosomatic medicine, 64, pp.469476 (2002)

18. W. Choi, C. Pantofaru\& S. Savarese, A general framework for tracking multiple people from a moving camera, PAMI, 35, pp.1577-1591 (2012)

19. M. D. Breitenstein, F. Reichlin et al, Robust tracking-by-detection using a detector confidence particle filter, ICCV, pp.1515-1522 (2009)

20. H. B. Shitrit, J. Berclaz et al,Tracking multiple people under global appearance constraints ICCV, pp.137-144 (2011)

21. P. Henry, M. Krainin et al, RGB-D mapping: Using Kinect-style depth cameras for dense $3 D$ modelling of indoor environments, IJRR, 31, pp.647-663 (2012)

22. K. Khoshelham\& S. Elberink, Accuracy and resolution of kinect depth data for indoor mapping applications, Sensors, 12, pp.1437-1454 (2012)

23. F. Grossi, V. Bianchi et al, Internet-based home monitoring and control, AAATE, pp.309-313 (2009)

24. O. Fuerst\& T. Fuerst, U.S. Patent Application No. $11 / 198,588(2005)$

25. A. Sharkey\& N. Sharkey, Granny and the robots: ethical issues in robot care for the elderly, Ethics and Information Technology, 14, pp.27-40 (2012)

26. CQC,Fundamental Standers, http://www.cqc.org.uk/ content/fundamental-standards, (accessed on December 2015).

27. CQC, About Us, http://www.cqc.org.uk/content /who-we-are, (accessed on December 2015).

28. CQC, Using surveillance: Information for providers of health and social care on using surveillance to monitor services (2015).

29. M. Boulos, N. Maged\& S. Wheeler, The emerging Web 2.0 social software: an enabling suite of sociable technologies in health and health care education. HILJ, 24, pp.2-23 (2007).

30. E. Machida, M. Cao et al, Human motion tracking of mobile robot with Kinect $3 D$ sensor, SICE, pp.22072211 (2012)

31. S. Huang \& J. Hong, Moving object tracking system based on camshift and Kalman filter,CECNet, pp.1423-1426 (2011) 